\title{
The black hole-bulge relations in active galactic nuclei
}

\author{
A. Wandel ${ }^{1}$ \\ ${ }^{1}$ The Hebrew University of Jerusalem, Israel
}

\begin{abstract}
We show that Massive Black Holes of AGNs follow the same approximately linear relation with the luminosity of the host spheroid (bulge) found in normal (quiescent) galaxies, with the black hole mass being 0.002 that of the bulge. We also demonstrate that Narrow line AGNs seem to have a significantly lower Mbh/Lbulge ratio, which implies a new strong correlation between $\mathrm{Mbh} /$ Lbulge and the width of the broad emission lines of AGNs (Wandel 2002). Combining existing data, new observations and extrapolation schemes based on the FaberJackson relation, the relation between the BH mass and the stellar velocity dispersion in the host bulge of AGNs is constructed. It too agrees with the relation found in quiescent galaxies.
\end{abstract}

\section{Introduction}

Massive Black Holes (MBHs) detected in the centers of many nearby galaxies (Kormendy and Richstone 1995, Kormendy \& Gebhardt (2002)) show an approximately linear relation with the luminosity of the host bulge, inferring the black hole mass is 0.001-0.002 of the bulge. In addition to those MBHs detected by techniques of stellar and gas kinematics, the masses of about three dozen MBHs in AGNs have been estimated by reverberation mapping of the broad emission-line region. High quality reverberation data and virial BH mass estimates are presently available for 20 Seyfert 1 nuclei (Wandel, Peterson and Malkan 1999, hereafter WPM) and 17 PG quasars (Kaspi et al. 2000), recently reanalysed by Peterson et al. (2004). The virial estimate has been shown to be consistent with the real BH mass (Peterson \& Wandel 1999; 2000). Previous work suggested that MBHs of active galactic nuclei (Seyfert galaxies and quasars) follow a similar relation (Laor 1998; Ho 1999; Wandel 1999). New and updated data for AGN confirm that AGNs and quiescent galaxies have the same BH-bulge mass relation (Wandel 2002; McLure \& Dunlop 2002). Several authors suggested that Narrow Line Seyfert 1s have lower MBH/bulge ratios (Wandel 1999;2002; Mathur Kuraskiewicz and Czerny 2001, Gruppe and Mathur 2003) however Botte et al. (2004) find in a different sample that NLS1s have the same BH-bulge relation as broad line AGNs. Ferrarese and Merritt (2000) and Gebhardt et al. (2000a) have found that MBH masses of inactive galaxies are better correlated with the stellar velocity dispersion in the bulge than with the bulge luminosity. Apparently this relation holds also for AGNs: the few Seyfert galaxies with stellar velocity data and reverberation $\mathrm{BH}$ mass estimates seem to be consistent with the $\mathrm{BH}$-velocity dispersion relation of inactive galaxies (Gebhardt et al. 2000b), a conclusion strengthened by observations of the velocity dispersion in reverberation mapped Seyfert galaxies (Ferrarese et al. 2001).

\section{The BH-bulge relation of AGNs}

We reexamine the BH-bulge mass relation of AGNs. We combine new and updated data to yield a data base of 55 AGNs (28 quasars, 18 Seyfert 1 galaxies and 9 narrow line 


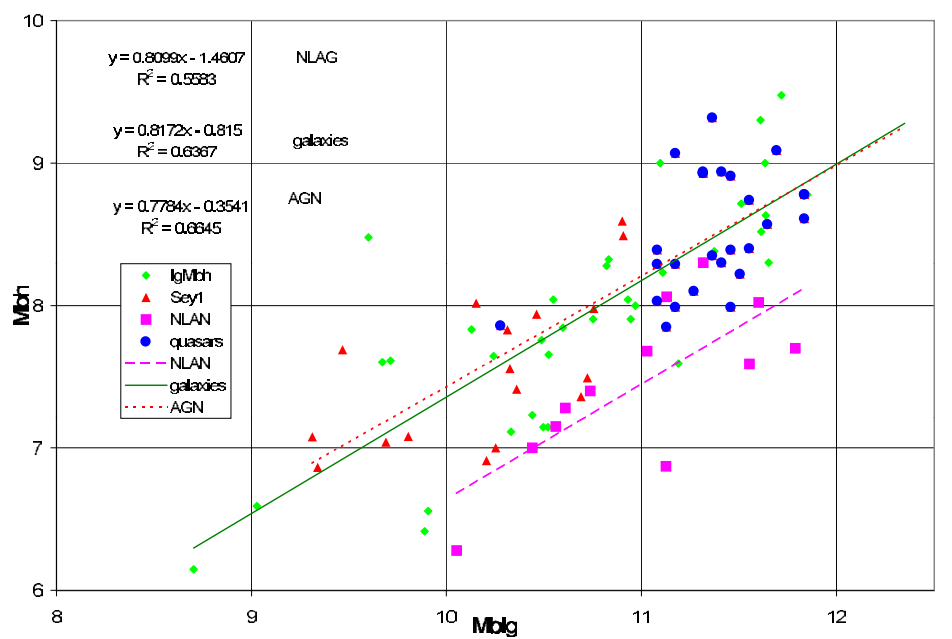

Figure 1. Black hole mass vs. bulge mass. Green diamonds represent quiescent galaxies, red triangles and blue circles are broad line Seyferts and quasars, respectively, pink squares are narrow line AGNs

Seyfert 1 galaxies and quasars). Most of these objects have reverberation mapping masses and almost all of them have the bulge luminosity measured directly by using bulge/disk decomposition (McLure \& Dunlop 2001). Our results (figure 1) reconfirm that broad-line AGNs follow the same BH-bulge relation as ordinary (inactive) galaxies, while narrow line AGNs have significantly lower BH/bulge mass ratios (Wandel 2002).

We find that broad line AGNs have an average black hole/bulge mass fraction of 0.0015 with a strong correlation $\left(M_{\mathrm{BH}} \propto L_{\text {bulge }}^{0.95 \pm 0.16}\right)$. This BH-bulge relation is consistent with the BH-bulge relation of quiescent galaxies and tighter than previous results (cf. Marconi and Hunt 2003). Narrow line AGNs (Narrow Line Seyfert 1s and quasars, defined by permitted lines narrower than $2000 \mathrm{~km} / \mathrm{s}$ ) appear to have a lower $\mathrm{BH} /$ bulge ratio, $M_{\mathrm{BH}} / M_{\text {bulge }} \sim 10^{-4}-10^{-3}$.

The lower BH/bulge ratio of narrow line AGNs may indicate that NLS1s have relatively smaller black hole masses. One way to check this possibility is the $\sigma-M_{B H}$ relation, but it appears that the narrow line Seyfert 1 galaxies with measured stellar velocity dispersion have similar $\sigma-M_{B H}$ ratios as broad line AGNs and quiescent galaxies (fig $4)$.

\section{Dependence on the Broad Emission Line Width}

The lower $\mathrm{BH} /$ bulge ratios of narrow-line AGN seems to be part of a continous trend: we find that the $M_{\mathrm{BH}} / L_{\text {bulge }}$ ratio in AGN is strongly correlated with the emission-line width, $M_{\mathrm{BH}} / L_{\text {bulge }} \sim F W H M(H \beta)^{2}$

This tight correlation may be related to the virial mass calculation $M_{\mathrm{BH}}=1.46 \times$ $10^{5} M_{\odot}\left(\frac{R_{b l r}}{\text { lt-days }}\right)\left(\frac{v_{F W H M}}{10^{3} \mathrm{~km} \mathrm{~s}^{-1}}\right)^{2}$ but given the $\mathrm{BH}$-bulge relation, we expect $M_{\mathrm{BH}} / L_{\text {bulge }}$ to be constant rather than strongly dependent on $F W H M(H \beta)$. Furthermore, combining the new relation with the virial relation we expect the dependence on the line width $\left(F W H M(H \beta)^{2}\right)$ to cancel and leave a scattered distribution. Rather, we find a new, 


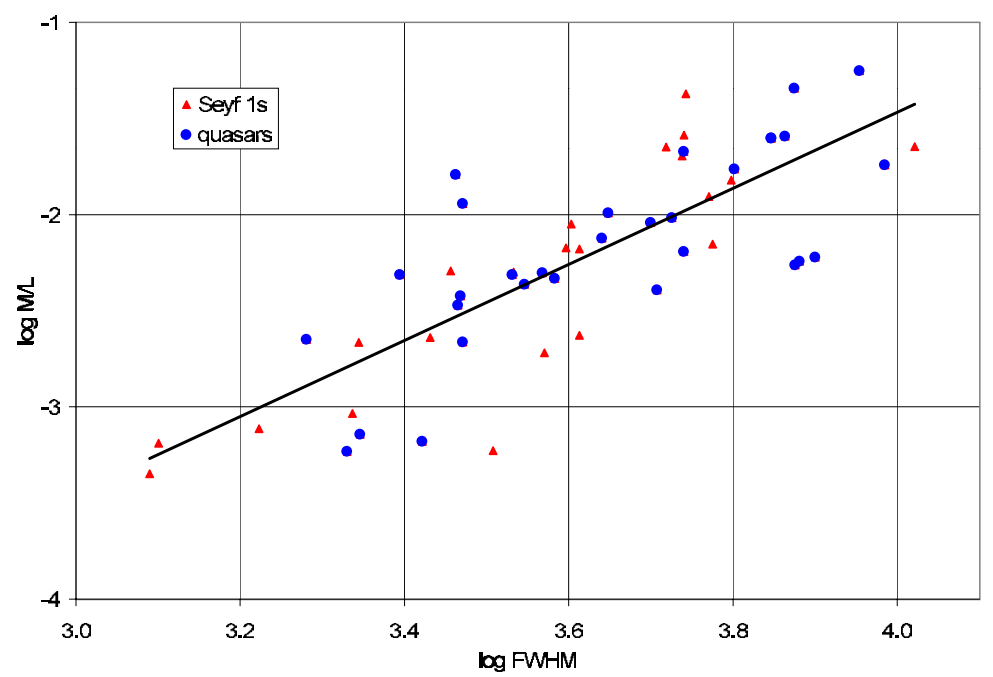

Figure 2. Black Hole Mass to bulge mass ratio vs. FWHM of $\mathrm{H} \beta$. Red triangles are broad line Seyferts and blue circles are quasars

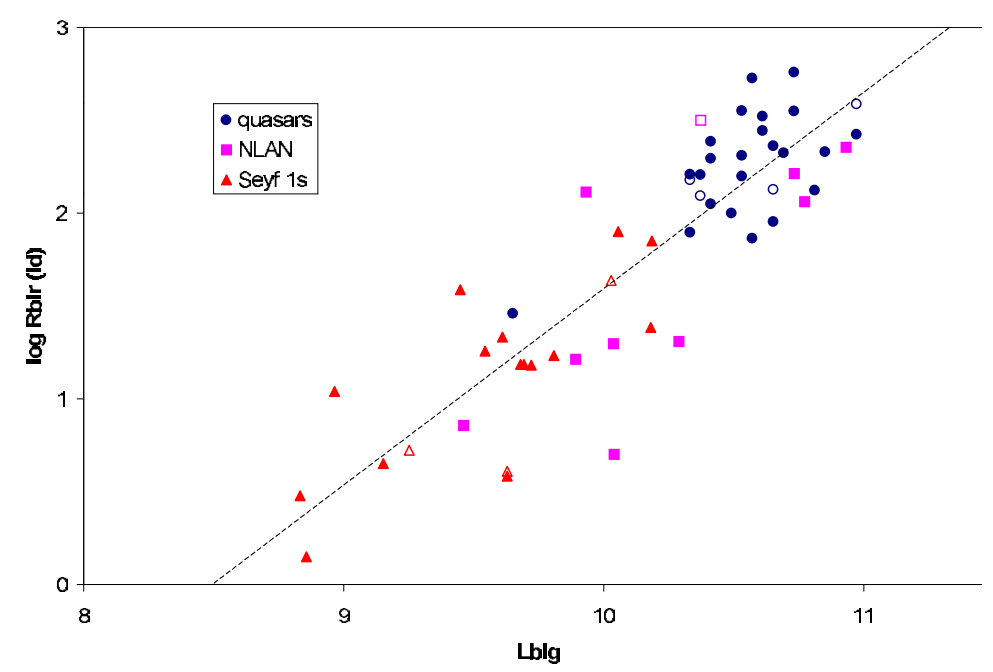

Figure 3. Size of broad emission-line region (BLR) vs. bulge luminosity. Triangles and circles are broad line Seyferts and quasars, respectively, squares are narrow line AGNs

strong nearly linear relation: a very tight correlation between bulge luminosity and BLR size (fig.3). The best fit of this correlation for all our sample is $R=13.5 L_{10}^{1.05} \quad$ lt - days with a correlation coefficient of 0.91 . This is a non trivial correlation, as it relates two independent observables: the bulge luminosity, a global galactic property on a scale of $\mathrm{kpc}$, and the distance of the line-emitting ionized gas from the central continuum source - the broad line region (BLR) size on a scale of few light days. 


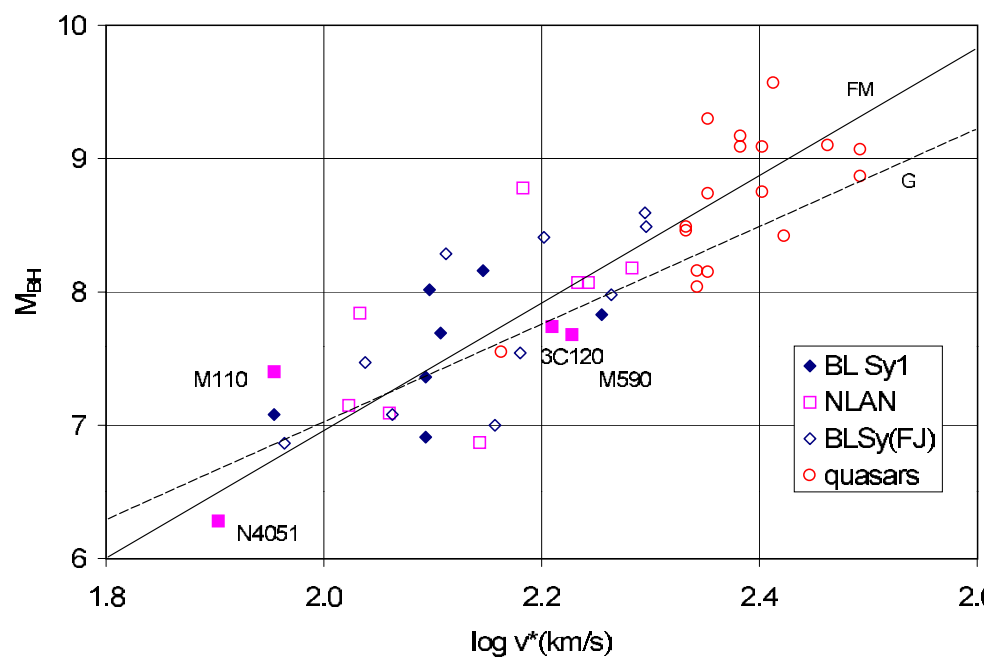

Figure 4. Black hole mass of AGNs plotted against the stellar velocity dispersion. Blue triangles are broad line Seyferts, pink squares denote NLS1s and red circles denote quasars. Solid symbols denote Seyferts with measured $\sigma$, open ones denote Seyferts for which $\sigma$ has been estimated from the Faber-Jackson relation (see text). The dashed and solid lines show the $\sigma-M_{\mathrm{BH}}$ relation of galaxies (Gebhardt et al. 2000a and Ferrarese \& Merritt 2000, respectively).

A possible explanation to this correlation may be as follows: it is known that the BLR radius scales with the AGN luminosity and the luminosity scales with the accretion rate. If the accretion rate is determined by the bulge size, the BLR size could be related to the bulge size. However, in the BLR-Lbulge relation narrow line AGNs do not seem to have a different scaling than broad line AGNs. This may indicate that the emission lines of NLANs appear narrowas a result of an inclination effect, for example, if the BLR has a flattened geometry and is viewed nearly edge on.

\section{The BH mass - velocity dispersion relation in AGN}

We find that in AGNs with "narrow" boad lines the black hole /bulge ratio seems to be systematically smaller than in broad line AGNs and in normal galaxies. Is this the case also for the $M_{\mathrm{BH}-\sigma}$ relation? The bulge velocity dispersion is measured for only 11 Seyfert galaxies, of which four (Mrk 110, Mrk 590, 3C120 and NGC 4051) can be classified as narrow line Seyfert 1s .

In figure 4 the $\mathrm{BH}$ mass is plotted against the stellar velocity dispersion. We see that broad line AGNs are consistent with the $M_{\mathrm{BH}}-\sigma$ relation of inactive galaxies $\left(M_{\mathrm{BH}} \propto \sigma^{\alpha}\right.$, with $\alpha=3.5-5$; Gebhardt et al. 2000a; Merritt and Ferrarese 2001a)

The narrow line Seyfert 1s with measured $\sigma$ denoted by solid pink squares in figure 4 ) seem to follow the same $M_{\mathrm{BH}}-\sigma$ relation of quiescent galaxies and broad line Seyferts.

For AGNs without a direct velocity dispersion measurement the relation between the narrow line width and the velocity dispersion (Nelson 2000;Botte et al. ) may be used to estimate the velocity dispersion in the bulge. Interestingly, a tight linear relation seems to exist between virial mass given by the narrow [OIII] line and the BH mass in Seyfert galaxies (Wandel and Mushotzky 1986). 
Here we use a different method to estimate the velocity dispersion - the Faber-Jackson (F-J) relation, which is applicable for AGNs with measured or estimated bulge luminosity. The "standard" F-J relation is $L=L_{o} \sigma_{2}^{4}$, where $\sigma_{2}=\sigma / 100 \mathrm{~km} \mathrm{~s}^{-1}$ and $L_{o}$ is a luminosity coefficient determined by a linear fit with a slope of 4 to those Seyfert Galaxies in our sample which have measured stellar velocity dispersion, which gives $L_{o}=1.6 \times 10^{9} \mathrm{erg} \mathrm{s}^{-1}$. Note that most narrow line AGNs added in this manner (open pink squares in fig. 4) do not have lower $\mathrm{BH}$ masses than the value expected from the $\sigma-M_{\mathrm{BH}}$ relation

\section{Conclusion}

We show that narrow-line AGNs have lower $M_{\mathrm{BH}} / L_{\text {bulge }}$ ratio than broad line AGNs, and find a strong correlation between this ratio and the broad emission-line width. However, in the $M_{b h} / \sigma^{4}$ relation narrow line AGNs seem to be similar to broad line AGNs.

\section{Acknowledgements}

This work was supported by BSF under grant number 1999336.

\section{References}

Botte, V. Ciori, S., Rafanelli, P., \& Di Mille, F. 2004, astro-ph/0402627

Ferrarese, L., Pogge, R. W., Peterson, B.M., Merritt, D. , Wandel, A., \& Joseph, C. L. 2001, ApJ, 555, L79

Gebhardt, K., et al. 2000a, ApJ, 539 L13

Gebhardt, K., et al. 2000b, ApJ, 543, L5

Gruppe, D., \& Mathur, S. 2003, astro-ph/0312390

Ho, L. C. 1999, in Observational Evidence for Black Holes in the Universe, (ed. S. K. Chakrabarti) Dordrecht: Kluwer, 157

Kaspi, S., et al. 2000, ApJ, 533, 631

Kormendy, J., \& Richstone, D. 1995, ARA\&A, 33, 581

Kormendy, J., \& Gebhardt, K. 2001, in proc. of the 20th Texas Symposium, (eds. H.Martel \& J.C.Wheeler), in press (astro-ph/0105230)

Laor, A. 1998, ApJ, 505, L83

Mathur, S., Kuraskiewiecz, J., \& Czerny, B. 2001, New Astronomy 6, 321

Merritt, D., \& Ferrarese, L. 2001a, MNRAS, 320, L30

Merritt, D., \& Ferrarese, L. 2001b, ApJ, 547, 140

McLure, R. J., \& Dunlop, J. S. 2001, MNRAS, 327, 199

McLure, R. J., \& Dunlop, J. S. 2002, MNRAS, 331, 795

Nelson, C. H. 2000, ApJ, 544, L91

Peterson, B. M., \& Wandel, A. 1999a, ApJ, 521, L95

- 2000, ApJ, 540, L13

Peterson, B. M., et al. 2004, ApJ, submitted

Richstone, D., et al., Nature, 395, A14

Wandel, A. 1999, ApJ, 519, L39

Wandel, A. 2000, in Probing the Physics of AGN by Multiwavelength Monitoring, (eds. B. M. Peterson, P. S. Polidan \& R. W. Pogge), ASP 224, 365

Wandel, A. 2001, BAAS, 33, 898

Wandel, A. 2002, ApJ, 565, 780

Wandel, A., Peterson, B., \& Malkan, M. 1999, ApJ, 526, 579

Wandel, A., \& Mushotzky, R. F. 1986, ApJ, 306, L61 


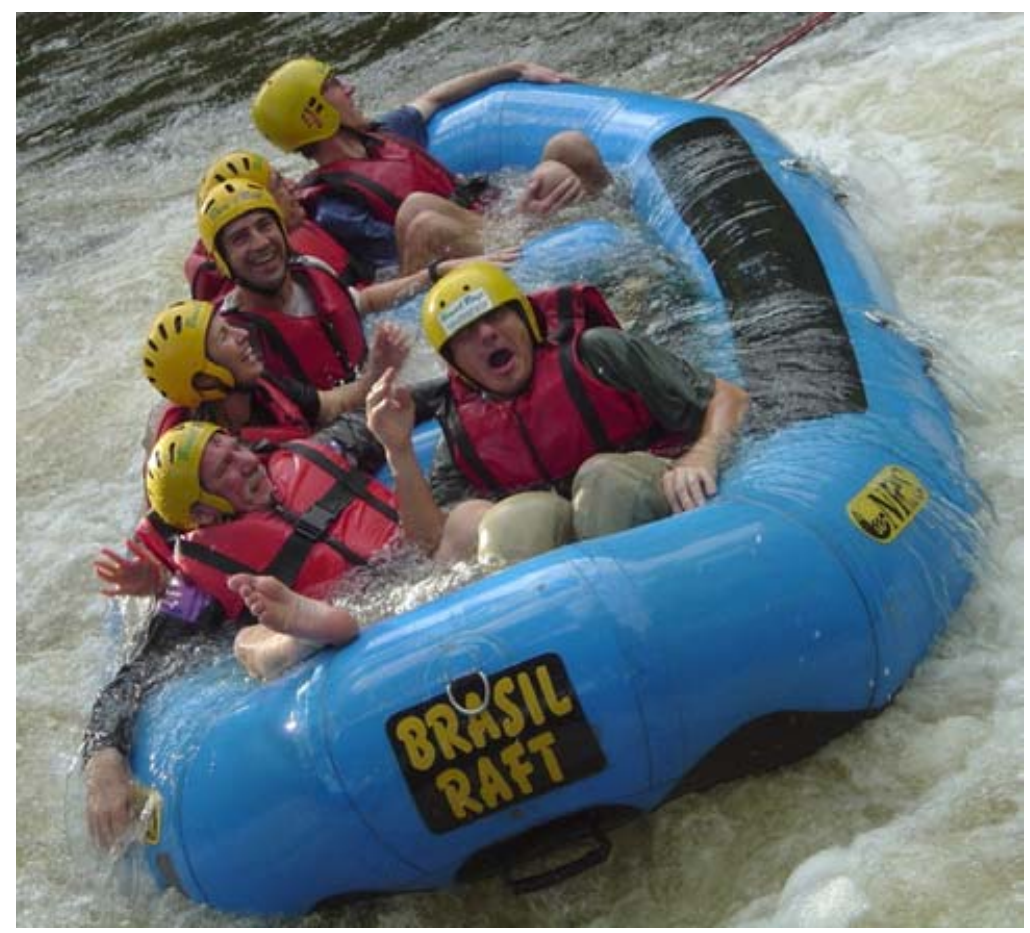

Rafting, from bottom to top: W. van Breugel, A. Filippenko, N. Edwards, A. Wandel, F. Hamman and J. Shields

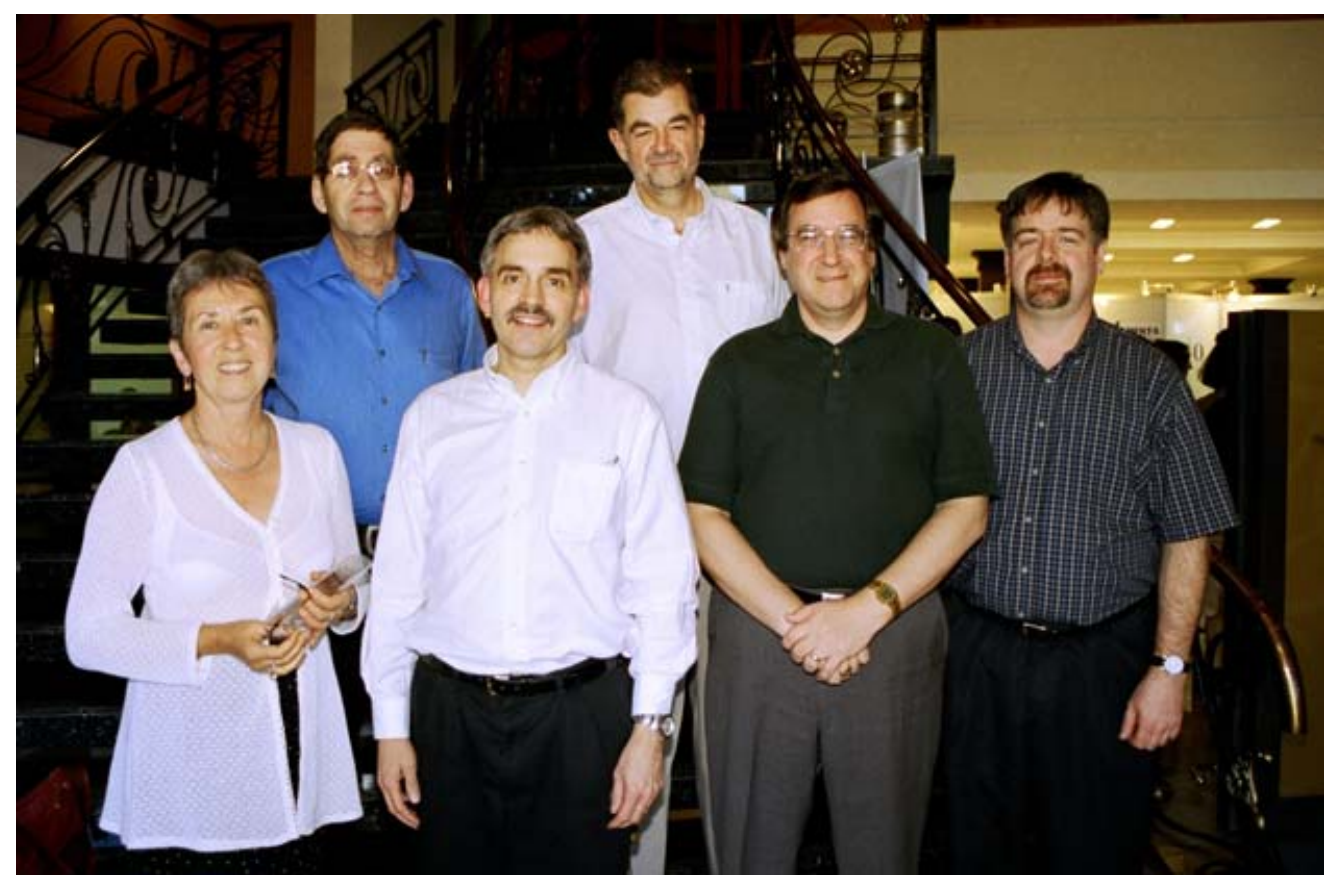

From left to right: D. Alloin, H. Netzer. B. Peterson, J. Clavel, G. Kriss and M. Crenshaw 